\title{
Computational Study of Ductile Sc-Intermetallics
}

\author{
P. BhardWAJ ${ }^{a}$, R. BhARDWAJ ${ }^{b, *}$ AND A.P. Mishra ${ }^{a}$ \\ ${ }^{a}$ Department of Physics, APS University, Rewa (MP), 468003, India \\ ${ }^{b}$ Department of Mathematics, Amity University, Kolkata (WB), 700135, India
}

(Received February 18, 2020; in final form March 25, 2020)

The electronic and elastic properties of PdSc and PtSc intermetallic compounds are reported in this paper. These intermetallics crystallize in the $\mathrm{CsCl}$ (B2-type) structure. The ground state properties such as lattice constant $a$, bulk modulus $B$, and its pressure derivative $B^{\prime}$ are calculated. These results show good agreement with the experimental and other theoretical results. The elastic constants were calculated. Besides we have studied Murnaghan's equation of state to obtain thermodynamical quantity. The present intermetallics confirm their ductile nature.

DOI: 10.12693/APhysPolA.137.1193

PACS/topics: intermetallic compounds, electronic properties, elastic properties

\section{Introduction}

From the past few years, the research of novel materials with specific properties and advanced application has attracted greater attention of metallurgists. There has been a continuing interest in the properties of the intermetallic compounds. Intermetallic compounds are the class of substances composed of definite proportions of two or more elemental metals. Intermetallics have properties and crystal structures that differ from their constituent metals. The ability of intermetallics to absorb hydrogen gas makes them potential candidates for hydrogen storage materials and intermetallic hydrides have been studied extensively for this purpose [1-5]. Jain et al. studied both the present compounds (PdSc and $\mathrm{PtSc}$ ) using full potential linearized augmented plane wave (FP-LAPW) method as implemented in the WIEN2k code. It is based upon density functional theory (DFT) within the generalized gradient approximation (GGA) in the scheme of Perdew, Burke and Ernzerhof (PBE). Wu and Cohen (WC) is used for the exchange and correlation effects [6].

According to the available literature it is initiated that extremely slight interest has been paid for electronic and elastic properties of PdSc and PtSc intermetallic compounds. A systematic study of electronic structure and elastic properties of $\mathrm{PdSc}$ and $\mathrm{PtSc}$ intermetallics have been presented in this paper. We have performed the Quantum Espresso code to study the structural and electronic properties of these intermetallics. These calculations are done for $\mathrm{PdSc}$ and $\mathrm{PtSc}$ compounds with $B 2$ structure using generalized gradient approximation (GGA) within density-functional theory (DFT). The basic ground state properties along with bulk modulus have been presented. Electronic band structure, density of states and some elastic properties are also investigated.

*corresponding author; e-mail: rkbhardwaj100@gmail.com
The ductile nature of these intermetallic compounds is predicted on the basis of calculated results. The aim of this work is to extend the understanding of anomalous ductility. The detailed methodology of presented calculation is given in the next section. In Sect. 2, a brief outline of the method of calculation is presented. In Sect. 3, the results are discussed.

\section{Methods of calculation}

Quantum Espresso is a computational technique for first principle calculation of periodic as well as disordered systems [7]. It is mainly based on DFT theory (electron-ion interaction), plane wave and pseudopotentials (electron-electron interaction). It calculates the ground state energy and Kohn-Sham orbital for both insulators and metals and various types of structural optimizations. Quantum Espresso can be used to study metals, semiconductors, as well as insulators. Three main components of method are plane wave self-consistent field (PWSCF), Car-Parinello (CP), first principle molecular dynamics (FPMD). For Quantum Espresso irreducible $k$ points are generated according to the Monkhorst-Pack scheme [8]. The Kohn-Sham single-particle functions were expanded on a basis of plane-wave set with a kinetic energy cut-off of $34 \mathrm{Ry}$. The Brillouin zone was sampled with $12 \times 12 \times 12 k$-point mesh, in order to get well converged ground state energy. The exchange and correlation effects have been treated within the GGA. The lattice parameter, bulk modulus and pressure derivative of the bulk modulus were determined by the standard procedure of computing the total energy for different volumes and fitted to Murnaghan's equation of state [9].

\section{Result and discussion}

To find the equilibrium atomic structure of the crystal the minimization of total energy is performed using the first principles pseudopotential (PWSCF) method. The equilibrium lattice constants were obtained by minimizing the total energy. The parameters were used in the 
Ground state properties.

TABLE I

\begin{tabular}{l|c|c|c|c|c|c}
\hline \hline & $a(\AA)$ & $B$ & $B^{\prime}$ & $C_{11}$ & $C_{12}$ & $C_{44}$ \\
\hline \multicolumn{7}{c}{$\mathrm{PdSc}$} \\
\hline this work & 3.295 & 106.87 & 4.22 & 148.67 & 85.98 & 36.77 \\
exp. & $3.283^{a}$ & - & - & - & - & - \\
others & $3.313^{b}$ & $62.70^{b}$ & - & - & - & - \\
& $3.316^{c}$ & $106.63^{c}$ & $4.32^{c}$ & $159.78^{c}$ & $87.59^{c}$ & $39.51^{c}$ \\
& $3.269^{c}$ & $121.99^{c}$ & $4.72^{c}$ & $153.39^{c}$ & $108.68^{c}$ & $64.00^{c}$ \\
\hline this work & 3.255 & 132.99 & 4.40 & 179.89 & 109.54 & 43.66 \\
exp. & 3.270 & - & - & - & - & - \\
others & $3.304^{b}$ & $80.26^{b}$ & - & - & - & - \\
& $3.305^{c}$ & $153.08^{c}$ & $4.52^{c}$ & $187.38^{c}$ & $113.31^{c}$ & $47.11^{c}$ \\
& $3.364^{c}$ & $148.28^{c}$ & $4.95^{c}$ & $186.80^{c}$ & $132.75^{c}$ & $58.85^{c}$ \\
\hline
\end{tabular}

${ }^{a}$ Refs. [10, 11], ${ }^{b}$ Ref. [12], ${ }^{c}$ Ref [6]

Elastic properties.

TABLE II

\begin{tabular}{c|c|c|c|c|c|c}
\hline \hline Solid & $G$ & $Y$ & $A$ & $\sigma$ & $C_{12}-C_{44}$ & $B / G$ \\
\hline PdSc & 34.49 & 93.42 & 1.71 & 0.35 & 49.21 & 3.09 \\
& $42.0^{a}$ & $113.2^{a}$ & $2.8^{a}$ & $0.34^{a}$ & $44.6^{a}$ & $2.94^{a}$ \\
\hline PtSc & 40.18 & 109.51 & 1.24 & 0.36 & 65.88 & 3.30 \\
& $43.0^{a}$ & $117.9^{a}$ & $2.1^{a}$ & $0.36^{a}$ & $73.9^{a}$ & $3.50^{a}$ \\
\hline
\end{tabular}

non self-consistent calculations for band structure. The energy values have been fitted to the Murnaghan equation of state [9] to obtain the equilibrium lattice constant $a$, bulk modulus $B$ and its pressure derivative $B^{\prime}$ at minimum equilibrium volume $V$. For this purpose, we use the following formula:

$$
P(V)=\frac{B}{B^{\prime}}\left[\left(\frac{V_{0}}{V}\right)^{B^{\prime}}-1\right],
$$

where the fit parameters are the equilibrium volume $V$, the bulk modulus $B$, given by

$$
B=-V \frac{\partial P}{\partial V}=V \frac{\partial^{2} E}{\partial V^{2}},
$$

and its derivative with respect to the pressure, $B^{\prime}=\mathrm{d} B / \mathrm{d} P$. In Table I the calculated structural parameters are tabulated. These parameters are compared with available results. It is clearly indicated that obtained results are in good agreement with the available experimental [10, 11] and other theoretical results [6, 12].

Further to calculate elastic properties the values of elastic constants $C_{11}, C_{12}$ and $C_{44}$ are computed and reported in Table I. In additional the computed values of Young modulus $Y$, isotropic shear modulus $G$, anisotropy parameter $A, B / G$ ratio, the value of $C_{12}-C_{44}$, and Poisson ratio, using $\mathrm{PWSCF}-\mathrm{GGA}$ approximations in $\mathrm{CsCl}$ structure have been given in Table II. All these parameters are comparable with others calculations [6, 10-12]. According to Pugh [13] if the value of $B / G>1.75$ the material behaves the ductile manner. The value of $B / G>1.75$ and $C_{12}-C_{44}>0$ confirm the ductile nature of both the intermetallics.

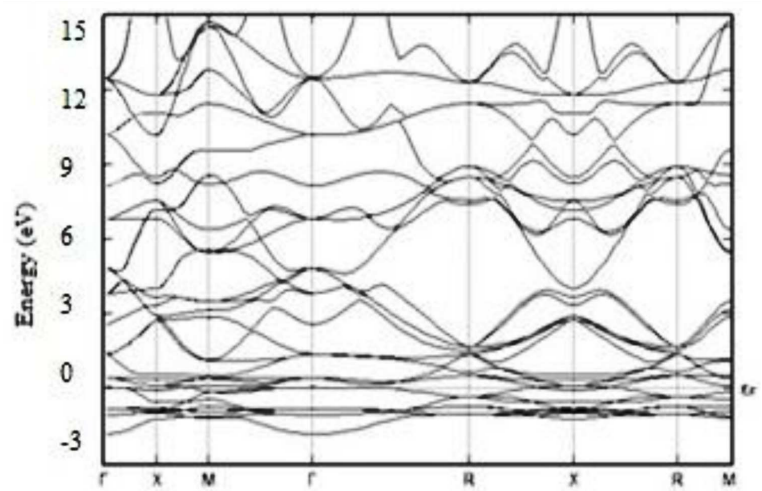

Fig. 1. Electronic band structure of PdSc in CsCl-phase.

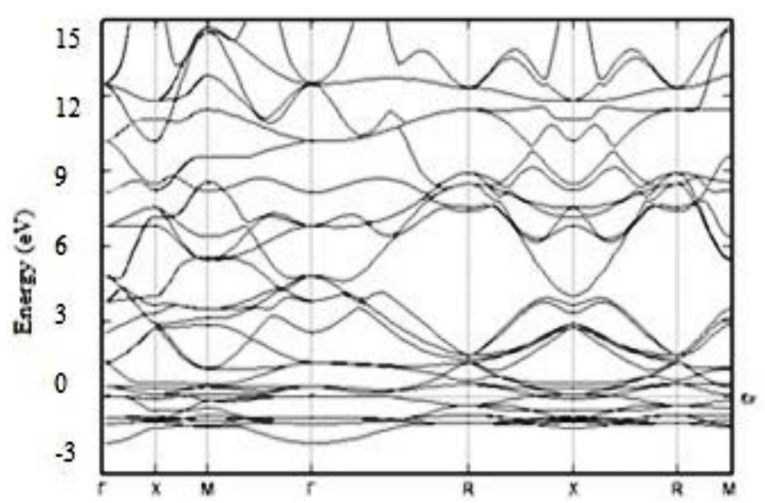

Fig. 2. Electronic band structure of PtSc in CsCl-phase.

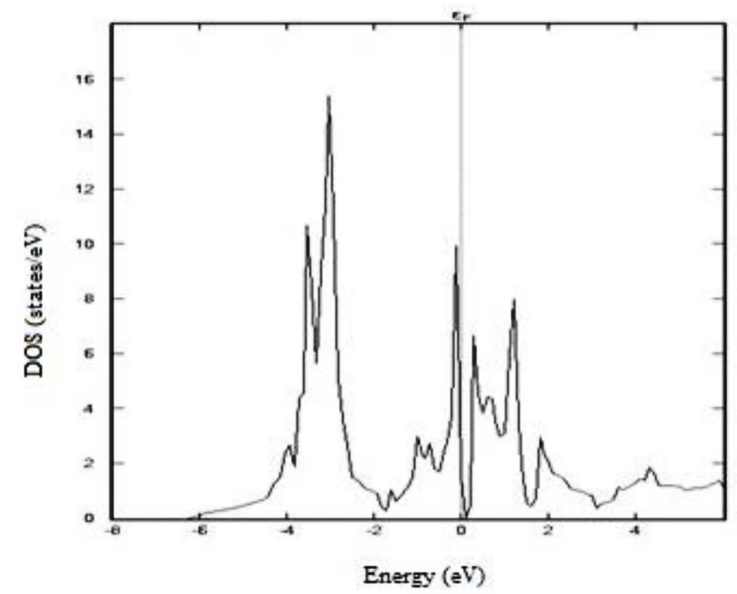

Fig. 3. Density of state (DOS) of PdSc in CsCl-phase.

Moreover, for investigating the electronic properties the electronic band structure (BS), total density of states (TDOS) and partial density of states (PDOS) of PdSc and PtSc are studied. These electronic properties are presented in Figs. 1-4, respectively. The band structure of PdSc is shown in Fig. 1 while Fig. 2 represents band structure of PtSc. In Figs. 3 and 4 the total density of 


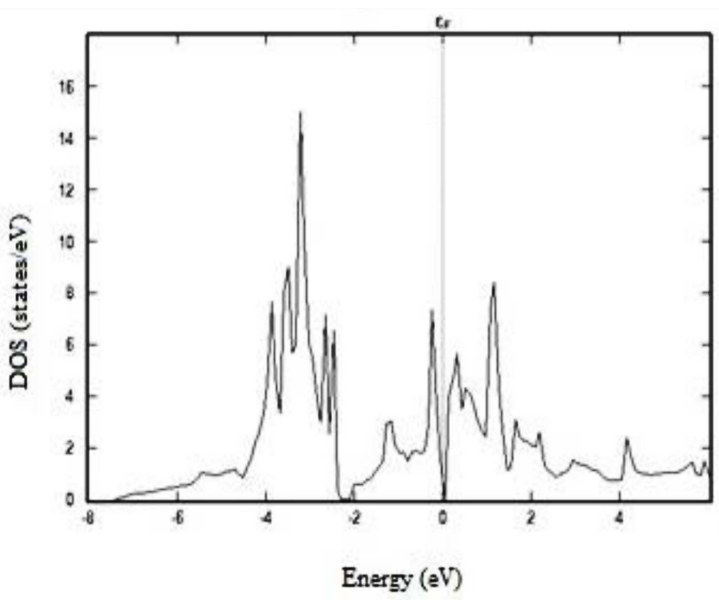

Fig. 4. Density of state (DOS) of PtSc in CsCl-phase.

states are plotted along with wave vector of both the compounds, respectively. Due to the hybridization it is clearly observed that both compounds show the metallic nature. Also, it is observed that $\mathrm{PtSc}$ has higher value of bulk modulus as compared to PdSc. So it is lighter than PdSc.

\section{Conclusion}

First principle calculation has been performed for studying the electronic study of present compounds in B2-type $(\mathrm{CsCl})$ phase. The lattice parameter and other fundamental properties are reported and compared with experimental data. Electronic band structure clearly shows the metallic behaviour of present compounds. Although elastic study clarifies that both intermetallics are ductile in nature.

\section{References}

[1] P. Kamakoti, D.S. Sholl, Phys. Rev. B 71, 9 (2005).

[2] Z. Zhang, A.M. Russell, S.B. Biner, K. Gschneidner, C.C.H. Lo, Intermetallics 13, 559 (2005).

[3] N.S. Stoloff, C.T. Liu, S.C. Deevis, Intermetallics 8, 1313 (2000).

[4] Y.J. Shi, Y.L. Du, G. Chen, G.L. Chen, Mater. Trans. 49, 2480 (2008).

[5] S.V. Meschel, P. Nash, Xing-Qiu Chen, J. Alloys Comp. 492, 105 (2010).

[6] E. Jain, G. Pagare, S. Singh Chouhan, S.P. Sanyal, Adv. Phys. Theor. Appl. 19, 49 (2013).

[7] P. Giannozzi, S. Baroni, N. Bonini, et al., J. Phys. Condens. Matter 21, 395502 (2009).

[8] P. Giannozzi, C. Cavazzoni, Il Nuovo Cimento 32, 49 (2009).

[9] F.D. Murnaghan, Proc. Natl. Acad. Sci. USA 30, 244 (1944).

[10] R.P. Elliott, Constitution of Binary Alloys, McGrawHill, New York 1968, Vols. 1-2.

[11] F.A. Shunk, Constitution of Binary Alloys, McGrawHill, New York 1971, Vols. 1-2.

[12] M.A. Baranov, Electron. Phys. Technol. J. 1, 49 (2006).

[13] S.F. Pugh, Philos. Mag. 45, 823 (1954). 